\title{
CRESCIMENTO PRÓ-POBRE NOS ESTADOS BRASILEIROS: ANÁLISE DA CONTRIBUIÇÃO DA CONSTRUÇÃO CIVIL USANDO UM MODELO DE DADOS EM PAINEL DINÂMICO, 1985-2008
}

\author{
Cristiano Ponzoni Ghinis * \\ Adelar Fochezatto ${ }^{\dagger}$
}

\begin{abstract}
Resumo
Este estudo analisou os efeitos da construção civil sobre a redução da pobreza nos estados brasileiros. Para isso, foram estimados modelos de dados em painel dinâmico para todas as unidades da federação no período 1985-2008. Os resultados indicam que o crescimento da construção civil tem sido pró-pobre na maior parte dessas unidades tanto no curto quanto no longo prazo. Os resultados indicam também que os impactos do setor sobre a redução da pobreza foram maiores que os de outros setores analisados.
\end{abstract}

Palavras-chave: Construção civil; Crescimento pró-pobre; Dados em painel.

\begin{abstract}
This study examined the effects of construction on the reduction of poverty in the Brazilian states. For this, models were estimated in dynamic panel data for all units of the federation in the period 1985-2008. The results indicate that growth in construction has been pro-poor in most of these units in both the short and long term. The results also indicate that the impacts of industry on poverty reduction were higher than those of other industries analyzed.
\end{abstract}

Keywords: Construction; Pro-poor growth; Panel data.

JEL classification: $\mathrm{O} 18, \mathrm{C} 23$

\footnotetext{
* Prefeitura Municipal de Porto Alegre (DMAE/PMPA). E-mail: cristianoghinis@bol.com.br

† Pontifícia Universidade Católica do Rio Grande do Sul (PUCRS), bolsista do CNPq. E-mail: adelar@pucrs.br
} 


\section{Introdução}

Desde a "segunda geração de economistas do desenvolvimento", assim denominados por Meier (2001), o crescimento econômico deixou de ser visto como condição necessária e suficiente para a promoção do bem-estar social. Como refere Sen (1999), é crucial avaliar o crescimento não somente como um fim, mas também como um meio de expandir as liberdades desfrutadas pelos membros de uma sociedade. Nessa concepção, a análise do crescimento tem como base não a sua quantidade, mas a sua qualidade, uma vez que a ampliação da base de recursos de uma sociedade somente se traduz em desenvolvimento quando é capaz de melhorar a qualidade de vida de sua população. É essencial, portanto, analisar não apenas o quanto uma economia cresceu, mas como foi este crescimento e, por consequência, qual o impacto distributivo do mesmo. Conforme Ribeiro et al. (2004), essa mudança de ênfase na avaliação do impacto do crescimento econômico sobre a qualidade de vida dos indivíduos combina melhor a eficiência e a equidade na promoção do desenvolvimento humano.

Em linhas gerais, não há um consenso para a definição do crescimento própobre e a própria pobreza não é um fenômeno unidimensional, ligado estritamente à insuficiência de renda. Apesar das eventuais discrepâncias acerca do conceito de crescimento pró-pobre, pode-se entendê-lo como aquele crescimento capaz de habilitar os pobres a participarem da atividade econômica, fazendo com que o aumento generalizado da renda os beneficie proporcionalmente mais do que aos não pobres (Ribeiro et al. 2004).

Nessa perspectiva, diversos trabalhos têm apontado que a relação entre crescimento e redução da pobreza deve ser vista como uma relação empírica, altamente específica, para cada país ou mesmo região, uma vez que ela não pode ser definida a priori por argumentos essencialmente teóricos. Por exemplo, Datt \& Ravallion (1998) demonstraram que, para o caso da Índia, maiores níveis de produtividade agrícola contribuem significativamente para a redução da pobreza rural. Mas observaram também que o crescimento do PIB do setor não agrícola exerce efeitos heterogêneos sobre a redução da pobreza entre os estados indianos (Ravallion \& Datt 2002). Conforme os autores, o crescimento do setor não agrícola "[...] is more pro-poor in states with higher initial farm yields, higher female literacy rates, lower infant mortality, lower urban-rural disparities in consumption levels and lower initial landlessness." (Ravallion \& Datt 2002, p.393).

Desse modo, pode-se concluir que as condições de convertibilidade são dependentes do contexto no qual são analisadas, sendo possível explorar, ainda no plano teórico, um amplo conjunto de fatores que, conceitualmente, podem influenciar a conversão do crescimento econômico em redução da pobreza. Ribeiro et al. (2004) apresentam um resumo desses grupos de fatores, dentre os quais se destacam os padrões setoriais do crescimento e o grau de intensidade e uso dos fatores de produção. ${ }^{1}$

Com base nessa perspectiva mais ampla do crescimento econômico, um aspecto ainda pouco explorado na literatura empírica, ainda que se tenha uma ideia já bem difundida, é o perfil pró-pobre do crescimento dos setores, particularmente da construção civil, objeto deste estudo. A importância desse setor

\footnotetext{
${ }^{1}$ Para maiores detalhes ver Ribeiro et al. (2004).
} 
está no fato de que ele, em termos relativos, pode proporcionar a inclusão de pessoas com baixa qualificação e renda no mercado de trabalho.

Para analisar a construção civil sob a ótica do crescimento pró-pobre, o trabalho está organizado em cinco seções, além desta introdução. Na segunda seção analisam-se algumas características da construção civil que possam evidenciar o seu perfil pró-pobre em relação à geração de renda e emprego de baixa escolaridade. Em seguida, na terceira seção, mostram-se algumas medidas da evolução da pobreza no Brasil no período 1985-2008. Na quarta seção, são apresentados o modelo econométrico e os resultados encontrados. Por fim, são apresentadas as conclusões do estudo.

\section{Características do mercado de trabalho da construção civil}

Uma das características marcantes do processo produtivo da construção civil é a acentuada participação dos empregados de baixa escolaridade no total da força de trabalho do setor. Embora, nos últimos anos, tenha havido um aumento da demanda por mão de obra qualificada no setor, pode-se afirmar que, em termos relativos, ele continua empregando pessoas com baixa qualificação. ${ }^{2}$ Segundo informações da RAIS, a participação de empregados de baixa escolaridade ${ }^{3}$ no emprego total do setor vem sendo superior à verificada para o total das atividades produtivas. Mais precisamente, em 1985, tal participação era de $89,6 \%$ na construção civil e de $70,6 \%$ no total dos setores de atividade econômica. Em 2008, esses percentuais passaram, respectivamente, para $70,8 \%$ e $41,3 \%$. Na verdade, relativamente ao total dos setores produtivos, essa dependência aumentou no período, já que a queda de participação do emprego de baixa escolaridade no setor foi menor do que aquela verificada na totalidade das atividades econômicas. Com base nessas informações, pode-se constatar que permanece a particularidade do processo produtivo da construção civil de alta dependência do trabalho de baixa qualificação.

A Tabela 1 apresenta o emprego formal da construção civil e do total dos setores de atividade econômica, por níveis de escolaridade. Dentre as categorias que podem ser incluídas no trabalho de baixa escolaridade, a maior participação no total do emprego da construção civil foi a dos empregados com ensino fundamental completo. Porém, comparativamente ao total dos setores, destaca-se a maior participação relativa de empregados com até o 5 ano incompleto do ensino fundamental, que representava cerca de 2,5 vezes a participação desta mesma categoria para a totalidade das atividades produtivas.

Além disso, as participações das categorias de empregados com o 5 ao 9 ano incompleto do ensino fundamental também representavam relativamente mais que o dobro de participação na comparação com o total dos setores. Em resumo, pode-se observar no Brasil em todas as categorias uma maior participação relativa, com relação ao total das atividades econômicas, de empregados da construção civil com menores níveis de instrução, desde analfabetos até

\footnotetext{
${ }^{2} \mathrm{O}$ termo baixa qualificação, utilizado neste trabalho, refere-se ao baixo grau de instrução em função baixo número de anos de estudo dos empregados.

${ }^{3}$ Cabe lembrar que se optou por classificar como empregados de baixa escolaridade aqueles cujo grau de instrução fica restrito ao nível médio incompleto, pois na categoria de ensino médio completo são incluídos cursos técnicos profissionalizantes que poderiam qualificar, mesmo que de forma limitada, os empregados do setor. E os de alta escolaridade são aqueles com ensino médio completo até doutorado. Além disso, como está sendo utilizada a base de dados da RAIS, trata-se sempre do emprego formal.
} 


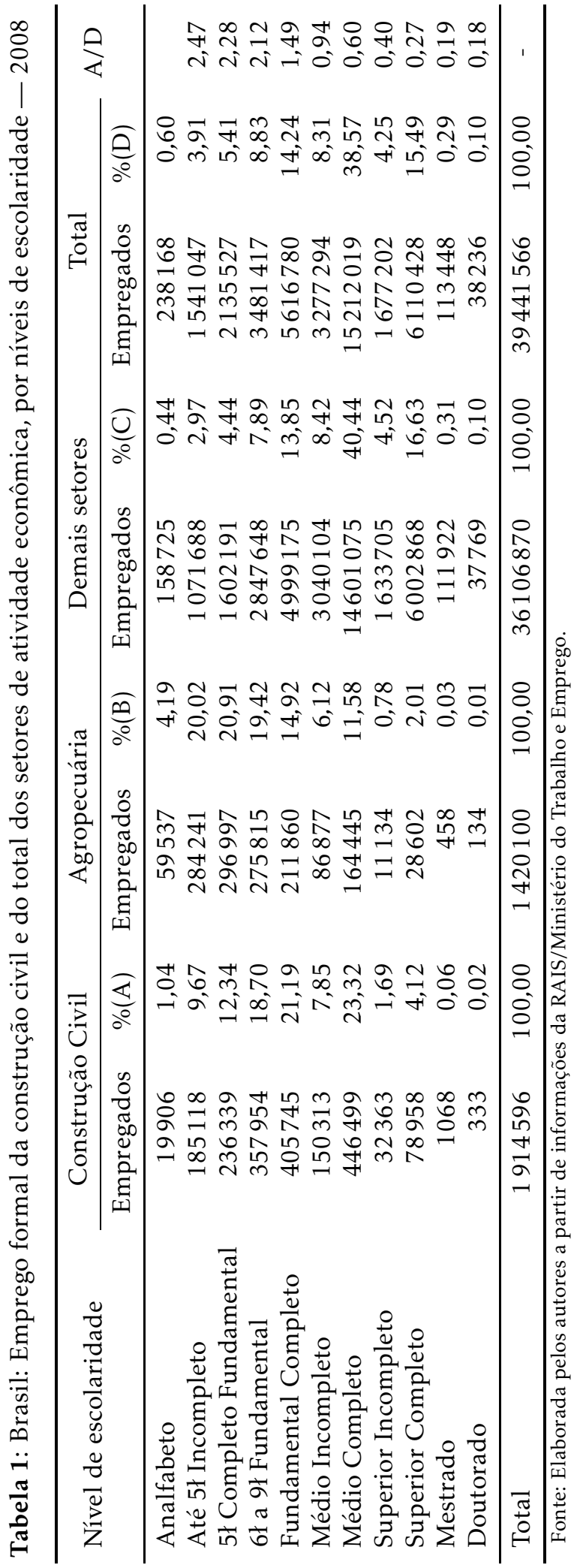


aqueles com ensino fundamental completo, além de uma menor participação relativa em todas as classes de empregados com maiores níveis de escolaridade (nota-se que, nesses casos, a razão entre as participações "A/B" é menor do que a unidade).

Além disso, conforme se observa na Tabela 2, para o total de empregados a construção civil apresentou uma média salarial de R\$1.114,67 em 2008, sendo inferior em $-17,9 \%$ na comparação com a média do total das atividades econômicas, que foi de $\mathrm{R} \$ 1.357,33$. No entanto, para o trabalho de baixa escolaridade, relativamente aos salários da mesma classe de empregados no total dos setores, o nível salarial da construção civil foi, em média, superior em $8,7 \%$. Mais precisamente, enquanto a média salarial dessa categoria de empregados para o total das atividades produtivas foi de $\mathrm{R} \$ 785,47$, a construção civil registrou um salário médio de $\mathrm{R} \$ 853,80$ em 2008 . Outro ponto a se considerar é que, dentre as classes que podem ser enquadradas no emprego de baixa escolaridade, todas apresentaram maiores salários relativos, comparativamente ao total dos setores, inclusive para os analfabetos.

Em suma, o crescimento do emprego formal da construção civil no Brasil está associado a dois aspectos relevantes: promove a inclusão de indivíduos com baixos níveis de instrução no mercado de trabalho; e para os empregados de baixa escolaridade, gera rendimentos superiores à média do total das atividades econômicas. Desse modo, pode-se afirmar que as particularidades do mercado de trabalho da construção civil enquadram-se, em boa medida, ao conceito de crescimento pró-pobre, como um crescimento que, em resumo, habilita os pobres a participarem da atividade econômica e a usufruírem de seus resultados.

\section{Evolução da pobreza no Brasil}

A pobreza é um fenômeno multidimensional, não ligado estritamente à insuficiência de renda. Ela abrange aspectos absolutos e relativos como, por exemplo, a fome, a doença, a vulnerabilidade econômica, a falta de moradia, a exclusão social, a impossibilidade de um nível de consumo satisfatório, a baixa escolaridade, dentre outros. Todavia, ainda no debate acadêmico e na orientação das políticas de combate a pobreza, predominam as medidas quantitativas (ou monetárias) de mensuração da mesma, tais como as linhas de pobreza. Dentre as principais justificativas para tanto, destacam-se: o alto grau de correlação entre a insuficiência de renda e as demais privações associadas à pobreza; a indisponibilidade de estatísticas sociais; a dificuldade de agregação e de ponderação de diferentes dimensões sociais; e a necessidade de medidas homogêneas que possibilitem comparações entre diferentes regiões ou localidades (Comim \& Bagolin 2002).

Nessa perspectiva, mesmo reconhecendo o seu caráter multidimensional, este trabalho se restringe, como em grande parte dos estudos empíricos, ao uso de medidas monetárias da pobreza, ligadas à insuficiência de renda. ${ }^{4}$ Especificamente, utiliza-se a metodologia de linhas de pobreza desenvolvida pelo Instituto Nacional de Pesquisa Econômica Aplicada (IPEA), que determina uma cesta básica de alimentos que satisfaz os requisitos nutricionais em cada região brasileira. A linha de extrema pobreza é uma estimativa do valor de uma

\footnotetext{
${ }^{4}$ Para uma análise qualitativa da pobreza no Rio Grande do Sul ver, por exemplo, Comim \& Bagolin (2002).
} 


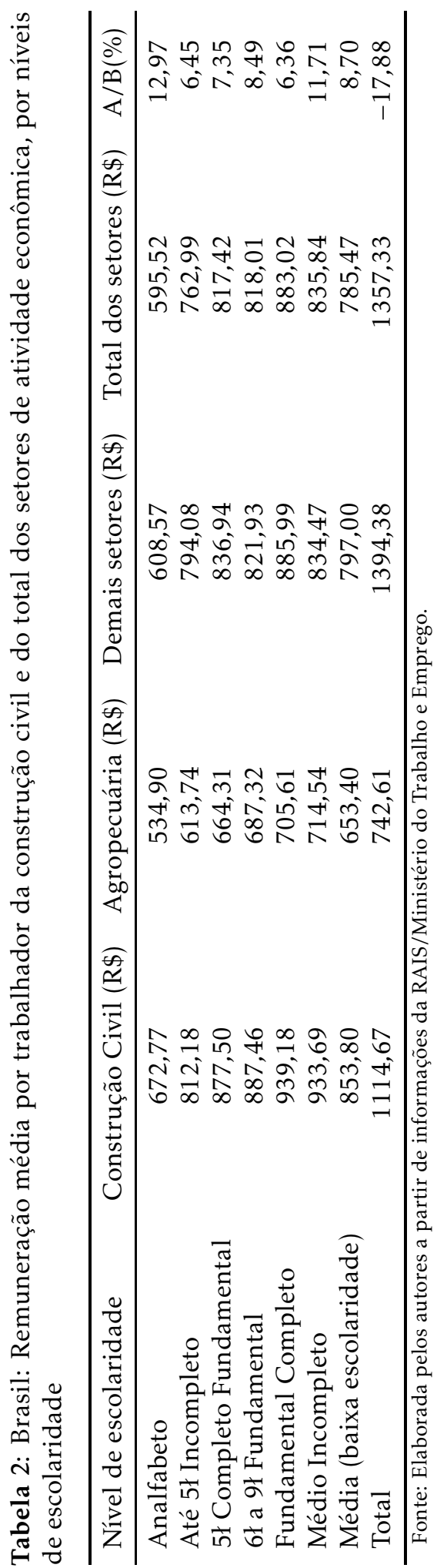


cesta de alimentos com o mínimo de calorias necessárias para suprir adequadamente uma pessoa numa determinada região, com base em recomendações da FAO (Organização das Nações Unidas para Agricultura e Alimentação) e da OMS (Organização Mundial da Saúde). E a linha de pobreza é considerada como o dobro do valor monetário desta linha de extrema pobreza.

A partir dessa metodologia, como já mencionado, a linha de pobreza é definida como uma renda mínima, sendo que indivíduos com renda abaixo deste mínimo são considerados todos como um grupo homogêneo de pobres. A divisão do número pelo total da população gera a tradicional medida de proporção de pobres. ${ }^{5}$ Segundo os dados da Pesquisa Nacional por Amostra de Domicílios (PNAD/IBGE) e de acordo com as linhas de pobreza do IPEA, a proporção de pobres diminuiu em praticamente todo o período em análise, exceto no final dos anos 80 , quando a economia brasileira sofria um processo inflacionário crônico, beirando a hiperinflação. Desse modo, enquanto a proporção de domicílios pobres era de $34,7 \%$ em 1985, esse percentual se reduziu, aproximadamente, pela metade no final dos anos em estudo, chegando a $17,2 \%$ em $2008 .^{6}$

Outro aspecto a se avaliar, de acordo com o conceito mais amplo de crescimento pró-pobre, é se a redução da pobreza tem sido acompanhada pela melhora distributiva da renda entre os indivíduos da sociedade. Nesse sentido, os dados da PNAD permitem dizer que os índices de Gini para a renda domiciliar per capita entre os indivíduos diminuiu simultaneamente ao processo de redução da pobreza no período em estudo, melhorando significativamente a distribuição de renda, principalmente a partir do início da década de 90. Portanto, nos anos em análise, a taxa de crescimento da renda dos pobres tem sido superior a taxa de crescimento da renda da população total. Assim, o processo de redução da pobreza que vem ocorrendo no país se enquadra, em boa medida, ao conceito mais abrangente de crescimento pró-pobre, que considera, também, a necessidade da melhora distributiva da renda entre a população.

A Tabela 3 mostra que, dentre os estados brasileiros, São Paulo e Rio Grande do Sul eram os dois estados com menor nível de pobreza do Brasil em 1992.7 Em 2008, essas posições foram ocupadas por Santa Catarina e São Paulo. No outro extremo, os dois estados com maior proporção de pobres eram Piauí e Paraíba em 1992 e Alagoas e Maranhão em 2008. Em linhas gerais, pode-se dizer que os estados da região Sul e Sudeste têm registrado os menores níveis de pobreza, os estados da região Centro-Oeste mantiveram-se em uma posição intermediária e que os estados das regiões Norte e Nordeste permaneceram entre os com maiores índices de pobreza. Estes, em termos de posições relativas, praticamente não mudaram sua situação no período em estudo. Desse modo, pode-se verificar que, durante praticamente duas décadas, o mapa da pobreza relativa entre os estados brasileiros, com algumas exceções, permaneceu praticamente inalterado. Isso mostra a grande dificuldade das regiões mais pobres (Norte e Nordeste) de saírem dessa condição, ainda que os níveis

\footnotetext{
${ }^{5}$ Cabe lembrar que se trata de uma medida de pobreza absoluta.

${ }^{6}$ É importante salientar que esses indicadores contemplam apenas as condições mínimas de sobrevivência dos indivíduos, particularmente relacionadas á alimentação. Assim, variações conceituais mais abrangentes em torno das diversas dimensões da pobreza, que incluíssem outras necessidades humanas básicas, poderiam ampliar estes dados de forma expressiva.

${ }^{7}$ Optou-se pela análise a partir desse ano porque foi quando, de acordo com os dados do IPEA (Gráfico 18), teve início de forma mais consistente o processo de redução da pobreza no País.
} 
de pobreza tenham se reduzido em todos os estados brasileiros.

Tabela 3: Proporção de domicílios pobres nos estados brasileiros $1992 / 2008$

\begin{tabular}{|c|c|c|c|c|c|}
\hline \multicolumn{3}{|c|}{1992} & \multicolumn{3}{|c|}{2008} \\
\hline $\begin{array}{l}\text { Unidade da Federa- } \\
\text { ção }\end{array}$ & $\begin{array}{l}\text { \% Domicí- } \\
\text { lios pobres }\end{array}$ & Posição & $\begin{array}{l}\text { Unidade da Federa- } \\
\text { ção }\end{array}$ & $\begin{array}{l}\% \text { Domicí- } \\
\text { lios pobres }\end{array}$ & Posição \\
\hline Piauí & 68,63 & 1 & Alagoas & 39,85 & 1 \\
\hline Paraíba & 62,76 & 2 & Maranhão & 38,24 & 2 \\
\hline Maranhão & 62,34 & 3 & Pernambuco & 37,17 & 3 \\
\hline Ceará & 61,05 & 4 & Piauí & 36,18 & 4 \\
\hline Tocantins & 59,77 & 5 & Paraíba & 33,60 & 5 \\
\hline $\begin{array}{l}\text { Rio Grande do } \\
\text { Norte }\end{array}$ & 59,64 & 6 & Bahia & 32,64 & 6 \\
\hline Pernambuco & 58,65 & 7 & Ceará & 31,57 & 7 \\
\hline Bahia & 57,09 & 8 & Sergipe & 31,07 & 8 \\
\hline Alagoas & 56,96 & 9 & $\begin{array}{l}\text { Rio Grande do } \\
\text { Norte }\end{array}$ & 27,69 & 9 \\
\hline Sergipe & 53,78 & 10 & Acre & 27,49 & 10 \\
\hline Pará & 48,68 & 11 & Amazonas & 26,96 & 11 \\
\hline Amazonas & 43,80 & 12 & Pará & 26,67 & 12 \\
\hline Amapá & 39,58 & 13 & Tocantins & 23,72 & 13 \\
\hline Rondônia & 38,90 & 14 & Roraima & 23,54 & 14 \\
\hline Paraná & 36,17 & 15 & Amapá & 21,56 & 15 \\
\hline Acre & 35,32 & 16 & Rondônia & 20,39 & 16 \\
\hline Mato Grosso & 32,77 & 17 & Rio de Janeiro & 11,45 & 17 \\
\hline Espírito Santo & 31,37 & 18 & Rio Grande do Sul & 10,72 & 18 \\
\hline Minas Gerais & 29,81 & 19 & Distrito Federal & 10,71 & 19 \\
\hline Goiás & 28,80 & 20 & Paraná & 10,31 & 20 \\
\hline Mato Grosso do Sul & 28,26 & 21 & Mato Grosso & 10,10 & 21 \\
\hline Roraima & 26,78 & 22 & Goiás & 9,94 & 22 \\
\hline Distrito Federal & 24,52 & 23 & Minas Gerais & 9,85 & 23 \\
\hline Santa Catarina & 24,00 & 24 & Mato Grosso do Sul & 9,29 & 24 \\
\hline Rio de Janeiro & 23,60 & 25 & Espírito Santo & 9,28 & 25 \\
\hline Rio Grande do Sul & 23,48 & 26 & São Paulo & 8,59 & 26 \\
\hline São Paulo & 19,96 & 27 & Santa Catarina & 5,87 & 27 \\
\hline Brasil & 34,96 & & Brasil & 17,17 & \\
\hline
\end{tabular}

\section{Construção civil e crescimento pró-pobre}

Esta seção tem por objetivo testar a hipótese de que o crescimento do emprego formal da construção civil tem sido pró-pobre. Para isso é construído um modelo de dados em painel dinâmico.

\subsection{Metodologia}

Para testar a hipótese de que o crescimento do emprego formal da construção civil tem sido pró-pobre, foram utilizadas informações do emprego formal da RAIS; da proporção de domicílios pobres e de indicadores de distribuição de renda do IPEA; dos gastos do governo com educação da Secretaria do Te- 
souro Nacional do Ministério da Fazenda; e de índices gerais de preços das Contas Regionais do Brasil/IBGE. Esses dados são coletados para todos os estados brasileiros no período 1985-2008, com um corte seccional, portanto, de 27 unidades geográficas e, temporal, de 24 anos. Desse modo, é construído um painel desequilibrado, com poucas lacunas em função da inexistência de informações nas bases de dados utilizadas, totalizando uma amostra de 638 observações para cada variável.

O modelo utilizado é especificado a partir da análise de trabalhos teóricos e empíricos que abordam a relação entre crescimento econômico e pobreza, como em Datt \& Ravallion (1998), Ravallion \& Datt (2002), Kakwani \& Pernia (2000), Ribeiro et al. (2004), Bezera et al. (2010) dentre outros. Utiliza-se a medida de pobreza em função do emprego formal dos setores agrícola e não agrícola, com o diferencial de desagregar setorialmente a construção civil. Além disso, a exemplo de Ribeiro et al. (2004), também se incluem como variáveis explicativas os gastos do governo com educação e a inflação. A primeira é utilizada como variável exógena de controle de políticas governamentais, que podem contribuir para a redução da pobreza. A segunda é usada porque, teoricamente, a alta dos preços prejudica proporcionalmente mais os pobres que os não pobres, ou seja, a inflação é, em princípio, pobreza-crescente. Além disso, inclui-se um indicador de distribuição de renda entre os indivíduos, contemplando, dessa forma, o conceito mais amplo de crescimento pró-pobre, que considera necessário que a redução da pobreza seja acompanhada pela melhora distributiva da renda, como em Kakwani \& Pernia (2000). Mais precisamente, busca-se estimar o modelo apresentado a seguir. ${ }^{8}$

$$
\begin{aligned}
\ln P 1_{i t}= & \beta_{0 i}+\beta_{1} \ln I G_{i t}+\beta_{2} \ln X 1_{i t}+\beta_{3} \ln X 2_{i t}+\beta_{4} \ln X 3_{i t} \\
& +\beta_{5} \ln G O V_{i t}+\beta_{6} \ln I N F_{i t}+\beta_{7} \ln I N F_{i t} * P R_{i t}+\mu_{i t}
\end{aligned}
$$

onde: $\ln =$ logaritmo natural das variáveis em análise; $i$ e $t=$ subscritos indicando que os valores das variáveis estão sendo observados no município " $i$ " e no ano " $t$ "; $P 1=$ proporção de domicílios pobres segundo a metodologia de linhas de pobreza desenvolvida pelo IPEA (apresentada na seção 3); $\beta_{0 i}=$ coeficiente de intercepto (ou constante) estimado para o estado " $i$ "; 9 IG = índice de Gini para a renda domiciliar per capita entre os indivíduos; $X 1=$ emprego formal do setor agrícola; $X 2=$ emprego formal do setor não agrícola; $X 3=\mathrm{em}$ prego formal da construção civil; GOV = gasto real per capita do governo com educação; ${ }^{10} I N F=$ índice geral de preços; $P R=$ variável dummy que assume valor um no período pós-Plano Real, isto é, a partir de $1994 .{ }^{11}$

\footnotetext{
${ }^{8} \mathrm{O}$ modelo é estimado através de mínimos quadrados ordinários (MQO), pois o teste de Hausman apontou para a escolha do método de efeitos fixos (ver Apêndice A).

${ }^{9}$ Esses coeficientes de intercepto estimados para cada estado a partir do método de efeitos fixos, mesmo sendo necessários para a estimação do modelo, não serão mais adiante reportados porque estão fora do escopo deste trabalho. Eles somente indicam o valor médio do logaritmo natural da medida de pobreza para cada estado quando as variáveis explicativas são iguais a zero.

${ }^{10}$ Corresponde ao gasto do governo com educação, dividido pelo número de habitantes (IBGE) e em relação aos índices de preços obtidos para cada estado (Contas Regionais/IBGE). Essa variável é considerada com uma defasagem de três anos (ou seja, para o período 1992-2005), primeiramente, em função da disponibilidade dos dados; e, em segundo lugar, porque os investimentos em educação somente geram retornos mais expressivos em termos de renda no médio ou longo prazo.

${ }^{11}$ Esse procedimento é necessário em função de uma quebra estrutural na tendência do nível geral de preços da economia brasileira a partir de então. Até 1993, o Brasil sofria com um processo
} 
É importante salientar que a utilização de um painel com séries temporais relativamente longas (de 24 anos), com um número proporcional de cortes seccionais (27 estados brasileiros), requer a realização de alguns testes adicionais de raiz unitária, para analisar se as séries são estacionárias. ${ }^{12}$ Nesse caso, os testes apresentados no Apêndice B.1 apontam que as séries em estudo são integradas de ordem $1(I(1))$, ou seja, são estacionárias somente em primeira diferença (por exemplo, $\ln X 3_{i t}-\ln X 3_{i t-1}$, ou $\Delta\left(\ln X 3_{i t}\right)$, para o caso do emprego formal da construção civil, onde os subscritos " $t$ " e " $t-1$ " indicam ano corrente e defasado em um período, respectivamente). Assim, pode-se utilizar o Mecanismo de Correção de Erros (MCE), que relaciona mudanças em uma variável a afastamentos do equilíbrio de longo prazo no período anterior. Todavia, essa estimação só faz sentido na existência de vetor(es) de cointegração, pois se as séries temporais não caminham juntas, os resíduos dessa regressão tendem a aumentar ao longo do tempo em valores absolutos. Isso significa que o resíduo será não estacionário ou integrado de ordem $1(I(1))$, de modo a se incorrer em regressão espúria. ${ }^{13}$ Em contraste, se o resíduo for estacionário, as séries serão cointegradas, o que, do ponto de vista econômico, significa que existe uma relação de equilíbrio, ou de longo prazo, entre as variáveis (Asteriou \& Hall 2007). Assim, apesar de individualmente as séries serem não estacionárias, elas são cointegradas indicando a existência de uma relação de longo prazo estável. Nesse aspecto, o teste de KAO dos resíduos para cointegração entre as séries do painel em estudo aponta para a existência de pelo menos um vetor de cointegração (Apêndice B.2), permitindo a estimação do MCE.

Dessa forma, a análise pode ser efetuada a partir de dados em painel dinâmico, sendo que os coeficientes do modelo (1) indicam as relações de equilíbrio de longo prazo entre as variáveis dependente e explicativas. Mais precisamente, o principal coeficiente de interesse, o $\beta_{4}$, corresponde à relação de longo prazo estimada entre o emprego formal da construção civil e a medida de pobreza. Isto é, a estimação de um coeficiente $\beta_{4}<0$ indica que o crescimento do emprego formal da construção civil tem sido pró-pobre no longo prazo; já um coeficiente $\beta_{4}>0$ aponta que tal crescimento tem sido empobrecedor; e, para o caso de $\beta_{4}=0$, os resultados passam a ser inconclusivos.

Além disso, a partir do MCE é possível estimar os efeitos de curto prazo e o coeficiente de ajuste do desequilíbrio de curto prazo na variável dependente ( $\ln P 1)$. O procedimento consiste em estimar um novo modelo com todas as variáveis do modelo (1) em primeira diferença (por exemplo, $\ln P 1_{i t}-\ln P 1_{i t-1}$, ou $\left.\Delta\left(\ln P 1_{i t}\right)\right)$, incluindo mais uma variável explicativa $\mu_{i t-1}$, que se trata dos resíduos estimados no modelo (1) defasados em 1 período (um ano). Mais especificamente, a equação é a seguinte:

inflacionário crônico, beirando a hiperinflação, que se iniciou na década de 80 . Mas, a partir de 1994, com a implementação do Plano Real e do subsequente sistema de metas inflacionárias, o país passou a registrar índices de inflação consideravelmente menores e relativamente mais estáveis ao longo dos anos.

${ }^{12}$ Quando uma série não é estacionária ela apresenta uma determinada tendência e suas médias e variâncias mudam ao longo do tempo.

${ }^{13}$ Regressão espúria se refere a uma estimação em que os resultados são espúrios, sem sentido, ou duvidosos. Ocorre quando uma relação entre duas ou mais séries temporais não correlacionadas simplesmente existe porque cada uma delas tem uma tendência, é uma série temporal I(1) ou ambas. 


$$
\begin{aligned}
\Delta\left(\ln P 1_{i t}\right)= & \beta_{0 i}+\beta_{1} \Delta\left(\ln I G_{i t}\right)+\beta_{2} \Delta\left(\ln X 1_{i t}\right)+\beta_{3} \Delta\left(\ln X 2_{i t}\right) \\
& +\beta_{4} \Delta\left(\ln X 3_{i t}\right)+\beta_{5} \Delta\left(\ln G O V_{i t}\right)+\beta_{6} \Delta\left(\ln I N F_{i t}\right) \\
& +\beta_{7} \Delta\left(\ln I N F_{i t} * P R_{i t}\right)+\delta \mu_{i t-1}+\varepsilon_{i t}
\end{aligned}
$$

onde: $\Delta=$ valores das variáveis em primeira diferença; $\mu_{i t-1}=$ resíduo estimado no modelo (1) para o estado " $i$ " no ano " $t-1$ ", isto é, defasado em um período (um ano); $\delta=$ coeficiente que indica a velocidade do ajustamento, ou seja, qual a proporção do desequilíbrio de curto prazo na variável dependente $(\ln P 1)$ que é corrigida a cada período (no caso, a cada ano) e $\varepsilon_{i t}=$ novo termo de erro (ou resíduo) estimado para o estado " $i$ " no ano " $t$ ". Cabe salientar que a interpretação para o $\beta_{4}$ é similar ao caso do modelo (1), porém indica a relação de curto prazo entre o emprego formal da construção civil e a medida de pobreza.

Após a realização desses procedimentos é possível a estimação das diferenças de sensibilidade nessa relação entre os estados brasileiros, já que esses efeitos podem ser heterogêneos. ${ }^{14}$ Para isso, é realizada uma regressão auxiliar semelhante ao modelo (1). A única diferença é a inclusão de uma variável dummy para cada estado em $\ln X 3$, que vai interagir com esta variável e gerar um vetor de $\beta_{4}$ 's composto pelos coeficientes estimados para cada unidade da federação. Desse modo, é possível analisar se o efeito do crescimento do emprego formal do setor sobre a redução da pobreza é significativo para cada um dos estados. O modelo alternativo para a estimação é o seguinte:

$$
\begin{aligned}
\ln P 1_{i t}= & \beta_{0 i}+\beta_{1} \ln I G+\beta_{2} \ln X 1_{i t}+\beta_{3} \ln X 2_{i t}+\beta_{4 i} \ln X 3_{i t} * D_{i} \\
& +\beta_{5} \ln G O V_{i t}+\beta_{6} \ln I N F_{i t}+\beta_{7} \ln I N F_{i t} * P R_{i t}+\mu_{i t}
\end{aligned}
$$

onde: $\beta_{4 i}=$ coeficiente angular estimado para o estado " $i$ " e $D_{i}=$ variável dummy que assume valor 1 para o estado " $i$ ".

\subsection{Resultados}

\section{Efeitos de curto prazo}

A Tabela 4 apresenta os resultados da estimação do modelo (2) para os efeitos de curto prazo. Observa-se, primeiramente, que o coeficiente de ajuste $\delta$, associado à variável $\mu_{i t-1}$, aponta para um ajustamento consideravelmente rápido, que é cerca de $48,5 \%$ da discrepância entre o valor efetivo (de curto prazo) e o valor de longo prazo de $\ln P 1$ eliminada a cada período. Em outras palavras, esse ajustamento é estabelecido em aproximadamente dois anos.

Com respeito à relação entre pobreza e distribuição de renda, nota-se que, no curto prazo, o coeficiente de ln IG estimado é positivo e significativo, ao nível de 5\% de significância. Pode-se constatar, assim, que a redução da pobreza no país está, de fato, estatisticamente relacionada com a melhora distributiva da renda entre os indivíduos nos anos 1985-2008.

Além disso, como propõe grande parte dos estudos teóricos e empíricos, como é o caso do trabalho de Datt \& Ravallion (1998) para a Índia, os resultados do modelo indicam que o crescimento do emprego formal do setor

\footnotetext{
${ }^{14} \mathrm{Na}$ estimação dos efeitos para cada estado a análise é efetuada somente em termos das relações de equilíbrio de longo prazo.
} 
agrícola tem sido pró-pobre no Brasil no curto prazo, e que a inflação tem sido pobreza-crescente no período em análise, ou seja, níveis altos de inflação têm prejudicado proporcionalmente mais os pobres que os não pobres. Em contraste, os resultados para o setor não agrícola não são conclusivos, com um coeficiente de curto prazo não significativo, mesmo ao nível de $10 \%$ de significância. ${ }^{15}$

Tabela 4: Resultados do modelo estimado de curto prazo

\begin{tabular}{lrrrr}
\hline Variável & Coeficiente & Erro-padrão & Estatística $t$ & Valor P \\
\hline$C$ & $-0,02575$ & 0,01272 & $-2,02425$ & 0,04340 \\
$\Delta(\ln I G)$ & 0,17044 & 0,06637 & 2,56801 & 0,01050 \\
$\Delta(\ln X 1)$ & $-0,18923$ & 0,03297 & $-5,73889$ & 0,00000 \\
$\Delta(\ln X 2)$ & 0,12690 & 0,08889 & 1,42769 & 0,15390 \\
$\Delta(\ln X 3)$ & $-0,27449$ & 0,04965 & $-5,52881$ & 0,00000 \\
$\Delta(\ln G O V)$ & 0,02709 & 0,00668 & 4,05413 & 0,00010 \\
$\Delta(\ln I N F)$ & 0,08848 & 0,01704 & 5,19195 & 0,00000 \\
$\Delta(\ln I N F * P R)$ & $-0,01241$ & 0,01358 & $-0,91393$ & 0,36110 \\
$\mu_{i t-1}$ & $-0,48477$ & 0,03570 & $-13,57981$ & 0,00000 \\
\hline \multicolumn{5}{c}{ Especificação dos efeitos: efeitos fixos (variáveis dummy) } \\
\hline R-quadrado & 0,34515 & & \\
R-quadrado ajustado & 0,30643 & & \\
Durbin-Watson & 1,71029 & & \\
Estatística $t$ & 8,91355 & & \\
Valor P (estatística $t)$ & 0,0000 & & \\
\hline Fonte: Elaboração dos autores.
\end{tabular}

Com relação ao caso específico da construção civil, verifica-se claramente que o crescimento do emprego formal do setor tem sido pró-pobre no Brasil no curto prazo. Chama a atenção, inclusive, a maior elasticidade da pobreza em relação ao emprego dessa atividade econômica, mesmo na comparação com o setor agrícola. Mais precisamente, considerando tudo o mais constante, o aumento de $1 \%$ no emprego formal do setor em estudo está associado à redução de $0,27 \%$ da proporção de domicílios pobres. Assim, a construção civil possui, em relação às demais atividades produtivas, o maior impacto sobre a redução da pobreza.

\section{Efeitos de longo prazo}

Em termos de efeitos de longo prazo, como se observa na Tabela 5, os resultados do modelo (1) apontam para uma maior sensibilidade na relação entre pobreza e distribuição de renda no país (o que pode ser visualizado pelo maior coeficiente de lnIG estimado, comparativamente à Tabela 4). ${ }^{16}$ Isso faz sentido na medida em que se espera que os efeitos distributivos da renda se façam perceber, de forma mais expressiva, principalmente no longo prazo.

\footnotetext{
${ }^{15}$ Além disso, como em Ribeiro et al. (2004), os resultados apontam que os gastos do governo com educação têm um impacto de aumento da pobreza.

${ }^{16} \mathrm{O}$ modelo é estimado com a correção de White cross-section standard errors and covariance, sendo que o teste formal para a existência de heterocedasticidade é apresentado no Apêndice C. Além disso, o Apêndice D apresenta o teste para multicolinearidade.
} 
No que se refere ao emprego formal do setor agrícola, ao contrário, observase que o impacto sobre a pobreza não é significativo. ${ }^{17}$ Nessa perspectiva, cabe relembrar o fenômeno recente mundial, enfatizado por Ribeiro et al. (2004), de metropolização da pobreza. Pois, se a tendência no Brasil é de aumento da pobreza fora das áreas rurais, o crescimento da agricultura não será necessariamente pró-pobre no longo prazo, como apontam os resultados do modelo (1). Efetivamente, os autores encontraram o mesmo resultado, não significativo estatisticamente, para a relação entre pobreza e renda agrícola no país, com base em dados para o período 1981-2002.

Com respeito à inflação, destaca-se que, para o total dos anos 1985-2008, ela tem sido pobreza-crescente também no longo prazo. Todavia, no período pós-Plano Real, observa-se uma relação inversa (pelo coeficiente negativo e significativo estimado de $\ln I N F * P R$ ). Isso se deve a uma quebra estrutural na tendência do nível geral de preços a partir de 1994. Quando o Brasil, em contraste à década de 80 , passou a registrar taxas de inflação mais moderadas, embora ainda positivas, concomitantemente ao processo de redução da pobreza que se consolidava no país. Desse modo, pode-se considerar o período 1994-2008 como um caso atípico no qual a inflação tem sido pró-pobre no Brasil. Porém, para a totalidade dos anos em estudo (1985-2008), taxas de inflação mais altas têm um impacto de aumento da pobreza, seja no curto ou no longo prazo.

Já o efeito do emprego do setor não agrícola tem sido pró-pobre no Brasil no longo prazo, ao nível de $10 \%$ de significância, com uma elasticidade pobreza-crescimento de $-0,13 \% .{ }^{18}$ No entanto, deve-se levar em conta a limitação desse resultado, já que pressupõe um padrão homogêneo nessa relação em todas as regiões do país. ${ }^{19}$ Neste aspecto, trabalhos recentes têm incluído dummies regionais para diferenciar as elasticidades da pobreza em relação ao crescimento nas diferentes regiões do Brasil, mesmo intraestaduais, como em Bezera et al. (2010), que considera as microrregiões de Alagoas.

De qualquer modo, em média, pode-se observar que a construção civil apresenta também o maior efeito sobre a redução da pobreza no longo prazo. Especificamente, o crescimento de $1 \%$ do emprego formal do setor em análise está relacionado, coeteris paribus, à redução de $0,20 \%$ da proporção de domicílios pobres. Com isso, as estimativas levam à conclusão de que, tanto no curto quanto no longo prazo, o crescimento do emprego formal do setor em estudo tem sido pró-pobre no país no período 1985-2008. E, além disso, o setor tem apresentado a maior elasticidade pobreza-crescimento, relativamente às demais atividades econômicas. Tais resultados se justificam, em grande parte, pela alta geração de emprego de pessoas com baixa instrução na construção civil, bem como pelos maiores salários relativos proporcionados para esses empregados do setor, na comparação com o total das atividades produtivas (como demonstrado na seção 3).

Entretanto, é necessário salientar, novamente, que esses efeitos podem ser heterogêneos entre os estados brasileiros, em função das acentuadas diferen-

\footnotetext{
${ }^{17}$ Bem como os gastos do governo com educação.

${ }^{18}$ Cabe lembrar que tal elasticidade se refere à variação percentual da proporção de domicílios pobres em função do aumento de $1 \%$ do emprego formal dos setores. Nesse caso, o crescimento de $1 \%$ do emprego formal do setor não agrícola está associado à redução de $0,13 \%$ da proporção de domicílios pobres.

${ }^{19}$ As diferenças de sensibilidade não são demonstradas, nesse caso, porque o trabalho é focado essencialmente na construção civil.
} 
ças socioeconômicas entre as regiões do Brasil.

Tabela 5: Resultados do modelo estimado de longo prazo

\begin{tabular}{lrcrc}
\hline Variável & Coeficiente & Erro-padrão & Estatística $t$ & Valor P \\
\hline $\mathrm{C}$ & 6,81911 & 0,74225 & 9,18705 & 0,00000 \\
$\ln I G$ & 0,21262 & 0,07262 & 2,92794 & 0,00350 \\
$\ln X 1$ & 0,06802 & 0,08253 & 0,82424 & 0,41013 \\
$\ln X 2$ & $-0,13881$ & 0,07840 & $-1,77052$ & 0,07710 \\
$\ln X 3$ & $-0,20478$ & 0,04686 & $-4,36988$ & 0,00000 \\
$\ln G O V$ & $-0,00138$ & 0,00244 & $-0,56324$ & 0,57350 \\
$\ln I N F$ & 0,08771 & 0,01774 & 4,94422 & 0,00000 \\
$\ln I N F * P R$ & $-0,06611$ & 0,02204 & $-3,00016$ & 0,00280 \\
\hline \multicolumn{5}{c}{ Especificação dos efeitos: efeitos fixos (variáveis dummy) } \\
\hline R-quadrado & 0,7513 & & \\
R-quadrado ajustado & 0,73771 & & & \\
Durbin-Watson & 1,03731 & & & \\
Estatística F & 55,29052 & & & \\
Valor P (estatística F) & 0,00000 & & & \\
\hline
\end{tabular}

Fonte: Elaboração dos autores.

Diferenças de sensibilidade entre os estados brasileiros

A Tabela 6 apresenta os resultados da estimação do modelo (3), que inclui as dummies regionais para diferenciar as elasticidades pobreza-crescimento da construção civil entre os estados brasileiros. Observa-se que, mesmo com as diferenças inter-regionais, o crescimento do emprego formal do setor em análise tem sido pró-pobre nos anos 1985-2008, na maior parte dos estados, mais precisamente, em 18 das 27 unidades da federação.

Nesse contexto, com os maiores efeitos estimados destacam-se Goiás, Minas Gerais e Rio de Janeiro, onde a redução da pobreza é mais que proporcional ao crescimento do emprego do setor em estudo (ou seja, as elasticidades pobreza-crescimento são maiores que a unidade). Por exemplo, em Goiás, o aumento de $1 \%$ do emprego formal da construção civil está associado, coeteris paribus, à redução de $1,26 \%$ da proporção de domicílios pobres.

Além disso, cabe relembrar que a elasticidade comum estimada para todos os estados brasileiros no modelo (1) foi de -0,20\%. Dessa forma, destacamse também pela elevada sensibilidade da pobreza em relação ao crescimento do emprego do setor em análise os estados do Espírito Santo (-0,90\%), Santa Catarina $(-0,86 \%)$, São Paulo $(-0,83 \%)$, Mato Grosso do Sul $(-0,78 \%)$ e Ceará $(-0,61 \%){ }^{20}$

Como já mencionado, estudos como o de Ravallion \& Datt (2002) enfatizam que as condições de convertibilidade do crescimento em redução da pobreza são dependentes do contexto no qual são avaliadas. Segundo os autores, nos estados indianos, observa-se que, em geral, o crescimento tem sido mais pró-pobre nas regiões que partem com melhores condições socioeconômicas iniciais. Em certa medida, isso se aplica aos resultados encontrados para os

\footnotetext{
${ }^{20}$ Os números entre parênteses correspondem às elasticidades pobreza-crescimento estimadas para cada caso (Tabela 6).
} 
Tabela 6: Resultados do modelo estimado com a especificação dos efeitos do emprego formal da construção civil por estado $\left(\beta_{4 i}\right)$

\begin{tabular}{|c|c|c|c|c|}
\hline Variável: $\ln X 3$ & Coeficiente $\left(\beta_{4 i}\right)$ & Erro-padrão & Estatística $t$ & Valor P \\
\hline Goiás & $-1,25584$ & 0,22632 & $-5,54902$ & 0,00000 \\
\hline Minas Gerais & $-1,25232$ & 0,19689 & $-6,36058$ & 0,00000 \\
\hline Rio de Janeiro & $-1,03524$ & 0,19871 & $-5,20973$ & 0,00000 \\
\hline Espírito Santo & $-0,90309$ & 0,09598 & $-9,40875$ & 0,00000 \\
\hline Santa Catarina & $-0,86388$ & 0,08625 & $-10,01607$ & 0,00000 \\
\hline São Paulo & $-0,82527$ & 0,11232 & $-7,34779$ & 0,00000 \\
\hline Mato Grosso do Sul & $-0,78026$ & 0,14835 & $-5,25957$ & 0,00000 \\
\hline Ceará & $-0,60529$ & 0,27204 & $-2,22496$ & 0,02650 \\
\hline Rio Grande do Sul & $-0,54111$ & 0,11791 & $-4,58899$ & 0,00000 \\
\hline Mato Grosso & $-0,48429$ & 0,14943 & $-3,24086$ & 0,00130 \\
\hline Piauí & $-0,46498$ & 0,15666 & $-2,96811$ & 0,00310 \\
\hline Pernambuco & $-0,45566$ & 0,17345 & $-2,62709$ & 0,00880 \\
\hline Paraíba & $-0,42216$ & 0,11362 & $-3,71570$ & 0,00020 \\
\hline Bahia & $-0,34241$ & 0,15226 & $-2,24886$ & 0,02490 \\
\hline Rio Grande do Norte & $-0,32786$ & 0,10144 & $-3,23215$ & 0,00130 \\
\hline Sergipe & $-0,25902$ & 0,08818 & $-2,93725$ & 0,00340 \\
\hline Rondônia & $-0,21050$ & 0,10875 & $-1,93564$ & 0,05340 \\
\hline Maranhão & $-0,14385$ & 0,08267 & $-1,74005$ & 0,08240 \\
\hline Acre & 0,17062 & 0,04974 & 3,43003 & 0,00060 \\
\hline Roraima & 0,37745 & 0,18997 & 1,98685 & 0,04740 \\
\hline \multicolumn{5}{|c|}{ Coeficientes não significativos, mesmo ao nível de $10 \%$ de significância } \\
\hline Paraná & $-0,50027$ & 0,55028 & $-0,90911$ & 0,36370 \\
\hline Tocantins & $-0,16385$ & 0,13932 & $-1,17609$ & 0,24000 \\
\hline Distrito Federal & $-0,13878$ & 0,15140 & $-0,91665$ & 0,35970 \\
\hline Pará & $-0,12149$ & 0,07808 & $-1,55591$ & 0,12030 \\
\hline Alagoas & $-0,09691$ & 0,15203 & $-0,63743$ & 0,52410 \\
\hline Amazonas & $-0,08105$ & 0,13294 & $-0,60970$ & 0,54230 \\
\hline Amapá & 0,15541 & 0,18105 & 0,85836 & 0,39110 \\
\hline \multicolumn{5}{|c|}{ Especificação dos efeitos: efeitos fixos (variáveis dummy) } \\
\hline R-quadrado & 0,80440 & & & \\
\hline R-quadrado ajustado & 0,78444 & & & \\
\hline Estatística F & 40,28930 & & & \\
\hline Valor P (estatística F) & 0,00000 & & & \\
\hline
\end{tabular}

Fonte: Elaboração dos autores. 
estados brasileiros, no caso específico da construção civil, pois a pobreza tem sido mais elástica ao crescimento em estados como Goiás, Minas Gerais, Rio de Janeiro, Espírito Santo, Santa Catarina, São Paulo, Mato Grosso do Sul e Rio Grande do Sul, os quais apresentaram, desde o início da década de 90, menores níveis de pobreza do que a média nacional.

Contudo, pode-se verificar, também, que o crescimento do emprego formal da construção civil tem sido pró-pobre mesmo em grande parte das regiões Norte e Nordeste, que, historicamente, registraram os piores indicadores de pobreza do país. Isso ocorre, em boa medida, em função do perfil pró-pobre do mercado de trabalho do setor supracitado, além de sua presença expressiva inclusive nessas regiões mais pobres. Em 2008, de acordo com informações da RAIS, as participações da construção civil no total do emprego formal das regiões Norte e Nordeste foram de, respectivamente, 5,5\% e 5,2\%; ao passo que, nacionalmente, tal participação foi de 4,9\%. Desse modo, o emprego da construção civil tem se localizado e contribuído significativamente para a redução da pobreza também em estados como o Ceará, Piauí, Pernambuco, Paraíba, Bahia, Rio Grande do Norte, Sergipe, Rondônia e Maranhão. ${ }^{21}$

\section{Considerações finais}

Este trabalho buscou analisar a construção civil sob o enfoque do crescimento pró-pobre, pressupondo que o setor pode ter contribuído para o processo de redução da pobreza no Brasil no período 1985-2008. Inicialmente, foi realizada uma revisão dos principais aspectos teóricos sobre tal crescimento. Assim, demonstrou-se que, embora recente, a literatura sobre o tema é ampla e controversa, mesmo quando se considera apenas a dimensão renda da pobreza. Conceitualmente, alguns autores o compreendem como aquele crescimento capaz de reduzir significativamente a pobreza, sem deixar clara o quão significativa é tal redução. Já outros levam em conta os aspectos distributivos da renda, considerando que, para o crescimento ser pró-pobre, a renda dos pobres deve crescer a taxas superiores às da renda da população total. Desse modo, a diminuição da pobreza estaria diretamente relacionada com a melhora distributiva da renda entre os indivíduos da sociedade. De qualquer forma, em resumo, pode-se entendê-lo como aquele crescimento capaz de habilitar os pobres a participarem da atividade econômica, fazendo com que o aumento generalizado da renda os beneficie proporcionalmente mais do que aos não pobres.

A partir desse conceito, verificou-se na literatura que a relação entre crescimento econômico e redução da pobreza é bastante complexa. Na prática, estudou-se que, dentre os diversos grupos de fatores que podem influenciar essa relação está o grau de intensidade e uso dos fatores de produção. Nesse aspecto, os melhores resultados na redução da pobreza podem estar associados a um padrão de crescimento que resulte na expansão relativamente alta do emprego de baixa qualificação, pois a redução da pobreza será mais elástica em relação ao crescimento quanto mais trabalho-intensivo for o mesmo, e, também, quanto maior o emprego de baixa qualificação gerado, ao menos

\footnotetext{
${ }^{21}$ De outra parte, os resultados são não conclusivos para o Distrito Federal, Paraná, Tocantins, Pará, Alagoas, Amazonas e Amapá. E o crescimento do emprego do setor tem sido empobrecedor nos estados do Acre e Roraima.
} 
no curto prazo, em decorrência da dificuldade de qualificar os pobres para o mercado de trabalho.

Nesse sentido, estimou-se que, em contraste ao observado para o total dos setores, o crescimento da construção civil é fortemente correlacionado com a geração de emprego de baixa qualificação no período estudado. Além disso, verificou-se que a construção civil também proporciona, para os empregados de baixa escolaridade, maiores salários relativos, na comparação com a totalidade das atividades econômicas. Dessa forma, o setor se enquadra, em boa medida, ao conceito de crescimento pró-pobre.

Para verificar se o emprego formal da construção civil tem sido pró-pobre, foram utilizadas informações predominantemente da RAIS e do IPEA para todos os estados brasileiros ao longo do período em estudo (1985-2008), construindo, assim, um painel dinâmico desequilibrado. Estimou-se, então, que o crescimento do emprego formal da construção civil tem sido pró-pobre tanto no curto quanto no longo prazo. Mais precisamente, no curto prazo, considerando tudo o mais constante, o aumento de $1 \%$ no emprego formal do setor em estudo está associado à redução de $0,27 \%$ da proporção de domicílios pobres. E, no longo prazo, estimou-se que esta elasticidade pobreza-crescimento é muito próxima, a saber, de $-0,20 \%$, isto é, o crescimento de $1 \%$ do emprego formal do setor em análise está relacionado, coeteris paribus, à queda de $0,20 \%$ da proporção de domicílios pobres.

Em suma, verificou-se que a construção civil possui, seja no curto ou no longo prazo, o maior impacto sobre a redução da pobreza em relação às demais atividades econômicas, mesmo quando se considera o emprego do setor agrícola. Além disso, apesar da heterogeneidade desses efeitos entre os estados brasileiros, observou-se que o crescimento do emprego na construção civil tem sido pró-pobre em 18 das 27 unidades da federação.

\section{Referências Bibliográficas}

Asteriou, D. \& Hall, S. G. (2007), Applied Econometrics, 1 edition edn, Palgrave Macmillan.

Bezera, L. A., Tejada, C. A. O., Santos, A. M. A. \& Jacinto, P. A. (2010), 'Própobre ou empobrecedor: Qual a contribuição do crescimento econômico para alagoas?', Análise 21(2), 162-174.

Comim, F. V. \& Bagolin, I. P. (2002), 'Aspectos qualitativos da pobreza no rio grande do sul', Ensaios FEE 23(especial), 467-490.

Datt, G. \& Ravallion, M. (1998), 'Farm productivity and rural poverty in india', The Journal of Development Studies 34(4), 62-85.

Kakwani, N. \& Pernia, E. (2000), 'What is pro-poor growth', Asian Development Review 16(1), 1-22.

Meier, G. M. (2001), The old generation of development economists and the new, in J. E. Meier, G. M.; Stiglitz, ed., 'Frontiers of development economics: the future in perspective', Oxford University Press.

Ravallion, M. \& Datt, G. (2002), 'Why has economic growth been more propoor in some states of india than others?', Journal of Development Economics 68(2), 381-400. 
Ribeiro, E. P., Comim, F. V. \& Porto Jr., S. S. (2004), Crescimento pró-pobre no brasil: uma análise exploratória, in 'XXXII Encontro Nacional de Economia - ANPEC, João Pessoa, Paraíba'. Trabalho apresentado na reunião anual da Associação Nacional dos Centros de Pós-Graduação em Economia, 2004.

Sen, A. (1999), Desenvolvimento como liberdade, 1 edição edn, Companhia das Letras.

\section{Apêndice A Escolha do modelo}

A.1 Teste F: escolha entre o método com constante comum e o modelo de efeitos fixos

$$
F=\frac{\frac{R_{E F}^{2}-R_{C C}^{2}}{N-1}}{\frac{1-R_{E F}^{2}}{N T-N-K}}=\frac{\frac{0,812}{26}}{\frac{0,249}{27 * 24-7-7}}=77,13
$$

onde: $R_{E F}^{2}=\mathrm{R}$-quadrado obtido no modelo de efeitos fixos; $R_{C C}^{2}=$ R-quadrado obtido no modelo de constante comum; $N=$ número de cortes seccionais (neste caso, 27 estados brasileiros); $T$ = número de períodos (isto é, 24 anos); $K=$ número de variáveis.

$$
F_{\text {calculado }}=77,13>F_{\text {critico }} \cong 1,79
$$

Rejeita-se $H_{0}$, mesmo ao nível de $1 \%$ de significância, e, portanto, o método de constante comum não é o mais adequado.

\section{A.2 Teste de Hausman: escolha entre o método de efeitos fixos e o modelo de efeitos aleatórios}

$H_{0}$ : estimadores do modelo de efeitos aleatórios são consistentes e eficientes $H_{a}$ : estimadores do modelo de efeitos aleatórios não são consistentes (embora eficientes)

$$
H=\left(\hat{\beta}_{E F}-\hat{\beta}_{E A}\right)^{\prime}\left[\operatorname{Var}\left(\hat{\beta}_{E F}\right)-\operatorname{Var}\left(\hat{\beta}_{E A}\right)\right]^{-1}\left(\hat{\beta}_{E F}-\hat{\beta}_{E A}\right) \approx \chi^{2}(k)
$$

onde: $\hat{\beta}_{E F}=$ coeficiente estimado no modelo de efeitos fixos; $\hat{\beta}_{E A}=$ coeficiente estimado no modelo de efeitos aleatórios; $\operatorname{Var}=$ variância; $\chi^{2}$ indica que a estatística H (Hausman) segue uma distribuição qui-quadrado.

$$
H_{\text {calculado }}=33,51>\chi_{\text {critico }}^{2} \cong 18,47
$$

Rejeita-se $H_{0}$, mesmo ao nível de $1 \%$ de significância, e, por conseguinte, os estimadores do modelo de efeitos aleatórios não são consistentes, isto é, deve-se optar pelo método de efeitos fixos. 
Tabela A.1: Resultados do teste de Hausman (H)

\begin{tabular}{|c|c|c|c|}
\hline Resumo dos resultados & Qui-quadrado (H) & Qui-quad. d.f. & Valor $\mathrm{P}(\mathrm{H})$ \\
\hline $\begin{array}{l}\text { Cross-section: efeitos } \\
\text { aleatórios }\end{array}$ & 33,50888 & 7,00000 & 0,00000 \\
\hline \multicolumn{4}{|c|}{ Comparações dos coeficientes estimados: } \\
\hline Variável & Efeitos fixos & Efeitos aleatórios & \\
\hline $\ln I G$ & 0,21262 & 0,19980 & \\
\hline $\ln X 1$ & 0,06802 & 0,07486 & \\
\hline $\ln X 2$ & $-0,13881$ & $-0,07820$ & \\
\hline $\ln X 3$ & $-0,20478$ & $-0,18634$ & \\
\hline $\ln G O V$ & $-0,00138$ & $-0,00319$ & \\
\hline $\ln I N F$ & 0,08771 & 0,08849 & \\
\hline $\ln I N F * P R$ & $-0,06611$ & $-0,06243$ & \\
\hline
\end{tabular}

Fonte: Elaboração dos autores.

\section{Apêndice B Testes de raiz unitária e de cointegração}

\section{B.1 Testes de raiz unitária para as séries em nível e em primeira diferença}




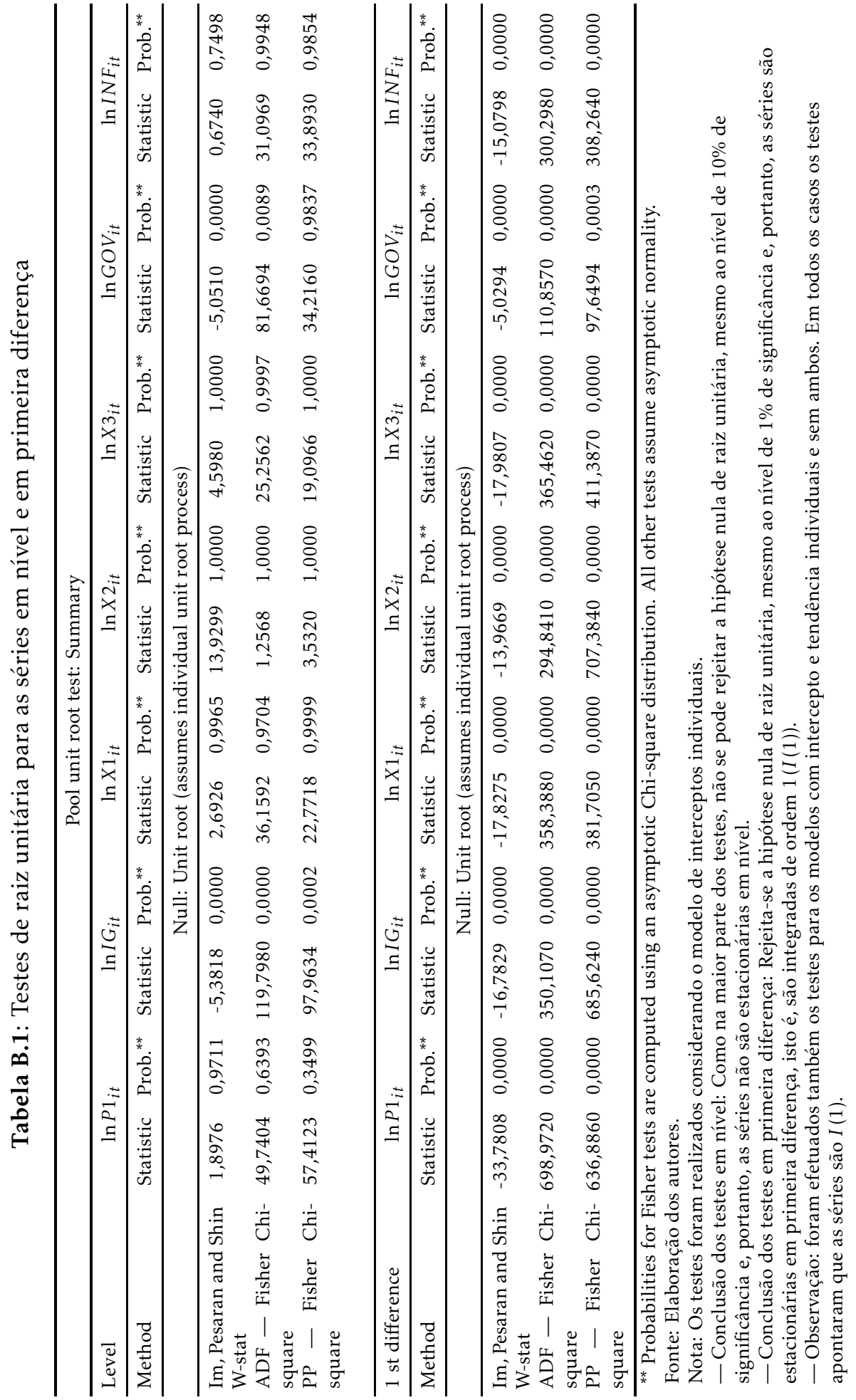




\section{B.2 Teste de KAO para cointegração}

Tabela B.2: Teste de KAO para cointegração

\begin{tabular}{|c|c|c|c|c|}
\hline \multicolumn{5}{|c|}{ Kao Residual Cointegration Test } \\
\hline \multicolumn{5}{|c|}{$\begin{array}{l}\text { Series: } \ln P 1_{i t}, \ln I G_{i t}, \ln X 1_{i t}, \ln X 2_{i t}, \ln X 3_{i t}, \ln G O V_{i t}, \ln I N F_{i t}, \\
\ln I N F_{i t} * P R_{i t} \\
\text { Null Hypothesis: No cointegration } \\
\text { Trend assumption: No deterministic trend } \\
\text { Lag selection: fixed at } 1 \\
\text { Newey-West bandwidth selection using Bartlett kernel }\end{array}$} \\
\hline & & & $\mathrm{t}$-Statistic & Prob. \\
\hline $\begin{array}{l}\text { ADF } \\
\text { Residual variance } \\
\text { HAC variance }\end{array}$ & & & $\begin{array}{r}-7,7203 \\
0,0687 \\
0,0335\end{array}$ & 0,0000 \\
\hline \multicolumn{5}{|c|}{ Augmented Dickey-Fuller Test Equation } \\
\hline \multicolumn{5}{|c|}{$\begin{array}{l}\text { Dependent Variable: D(RESID?) } \\
\text { Method: Panel Least Squares } \\
\text { Included observations: } 22 \text { after adjustments } \\
\text { Cross-sections included: } 27 \\
\text { Total pool (unbalanced) observations: } 583 \\
\end{array}$} \\
\hline Variable & Coefficient & Std. Error & $\mathrm{t}$-Statistic & Prob. \\
\hline $\begin{array}{l}\text { RESID?(-1) } \\
\text { D(RESID?(-1)) }\end{array}$ & $\begin{array}{l}-0,4638 \\
-0,1174\end{array}$ & $\begin{array}{l}0,0307 \\
0,0291\end{array}$ & $\begin{array}{r}-15,1225 \\
-4,0329\end{array}$ & $\begin{array}{l}0,0000 \\
0,0001\end{array}$ \\
\hline $\begin{array}{l}\text { R-squared } \\
\text { Adjusted R-squared } \\
\text { S.E. of regression } \\
\text { Sum squared resid } \\
\text { Log likelihood } \\
\text { Durbin-Watson stat }\end{array}$ & $\begin{array}{r}0,4279 \\
0,4269 \\
0,1683 \\
16,4482 \\
212,8222 \\
1,0125\end{array}$ & $\begin{array}{l}\text { Mean depe } \\
\text { S.D. depen } \\
\text { Akaike info } \\
\text { Schwarz cri } \\
\text { Hannan-Qu }\end{array}$ & $\begin{array}{l}\text { ndent var } \\
\text { dent var } \\
\text { criterion } \\
\text { iterion } \\
\text { uinn criter. }\end{array}$ & $\begin{array}{r}0,0128 \\
0,2223 \\
-0,7232 \\
-0,7082 \\
-0,7174\end{array}$ \\
\hline
\end{tabular}

Fonte: Elaboração dos autores.

Rejeita-se a hipótese nula de inexistência de cointegração, mesmo ao nível de $1 \%$ de significância e, por conseguinte, o teste aponta para a existência de pelo menos um vetor de cointegração.

\section{Apêndice C Teste LM Breusch-Pagan para heterocedasticidade}

\section{C.1 Regressão auxiliar}

$$
\begin{aligned}
\hat{u}_{i t}^{2}= & a_{1}+a_{2} \ln I G_{i t}+a_{3} \ln X 1_{i t}+a_{4} \ln X 2_{i t}+a_{5} \ln X 3_{i t} \\
& +a_{6} \ln G O V_{i t}+a_{7} \ln I N F_{i t}+a_{8} \ln I N F_{i t} * P R_{i t}+v_{i t}
\end{aligned}
$$

onde: $\ln I G_{i t} \ldots \ln I N F_{i t}=$ as variáveis explicativas do modelo original estimado na seção 4.1 ; $\hat{u}_{i t}^{2}=$ quadrado dos resíduos obtidos do modelo estimado na seção 4.1 (sem a correção de White). 
Tabela C.1: Variável dependente

\begin{tabular}{lrcrc}
\hline Variável & Coeficiente & Erro-padrão & Estatística $t$ & Valor P \\
\hline $\mathrm{C}$ & 4,01890 & 1,20590 & 3,33280 & 0,00090 \\
$\ln I G$ & $-0,23770$ & 0,11800 & $-2,01490$ & 0,04440 \\
$\ln X 1$ & 0,13930 & 0,05300 & 2,62810 & 0,00880 \\
$\ln X 2$ & $-0,55210$ & 0,12740 & $-4,33480$ & 0,00000 \\
$\ln X 3$ & 0,15790 & 0,07610 & 2,07420 & 0,03850 \\
$\ln G O V$ & $-0,00120$ & 0,00400 & $-0,31250$ & 0,75470 \\
$\ln I N F$ & 0,02210 & 0,02880 & 0,76830 & 0,44260 \\
$\ln I N F * P R$ & $-0,04060$ & 0,03580 & $-1,13390$ & 0,25730 \\
\hline R-quadrado & 0,10280 & & & \\
R-quadrado ajustado & 0,05380 & & & \\
Durbin-Watson & 2,12610 & & & \\
Estatística F & 2,09790 & & & \\
Valor P (estatística F) & 0,00040 & & & \\
\hline
\end{tabular}

Fonte: Elaboração dos autores.

\section{C.2 Formulação do teste de hipóteses}

$h_{0}: a_{1}=a_{2}=a_{3}=a_{4}=a_{5}=a_{6}=a_{7}=0$ (homocedasticidade)

$h_{a}$ : pelo menos um dos coeficientes é diferente de zero (heterocedasticidade)

\section{C.3 Cálculo da estatística LM e conclusão}

$$
L M=n R^{2}
$$

onde: $n$ = número de observações utilizadas para estimar a regressão auxiliar; $R^{2}=$ coeficiente de determinação da regressão auxiliar.

$$
L M=638 * 0,1028=65,61>\chi_{p-1, \alpha}^{2}=14,07
$$

Conclusão: rejeita-se $H_{0}$ ao nível de $5 \%$ de significância e, portanto, há evidências estatisticamente significativas da existência de heterocedasticidade.

\section{Apêndice D}




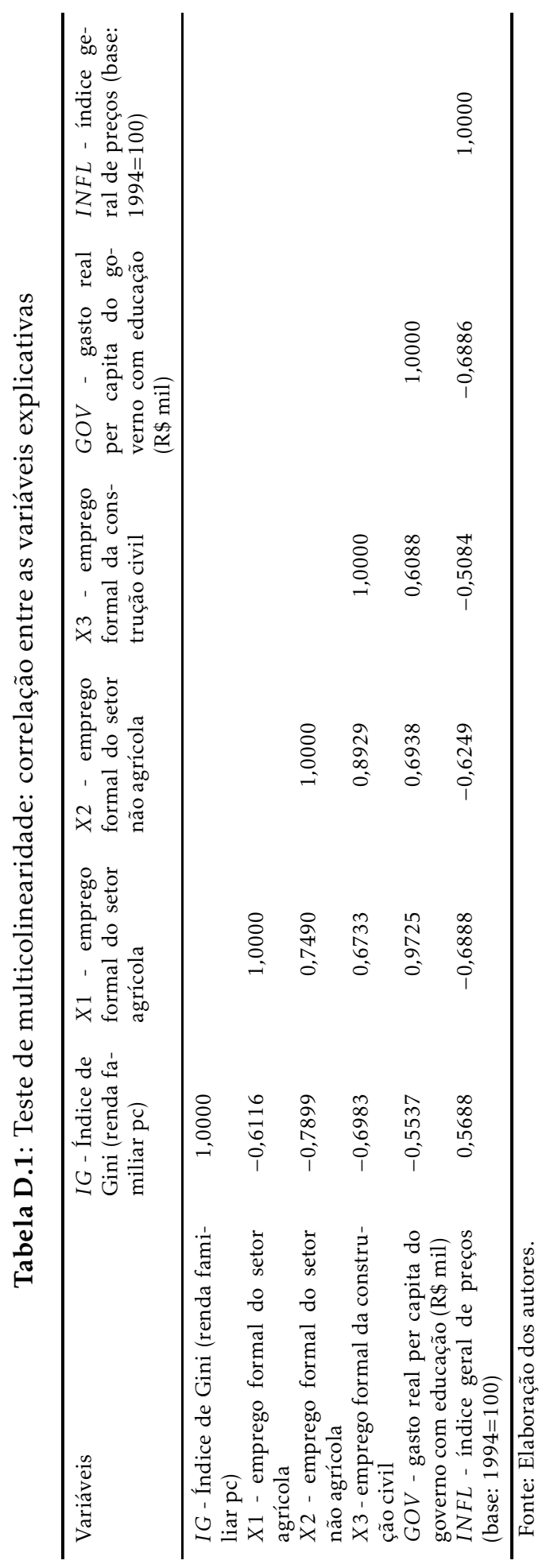

